\title{
Computations of the acoustically induced phase shifts of optical paths in acoustophotonic imaging with photorefractive-based detection
}

\author{
Florian J. Blonigen, Alex Nieva, Charles A. DiMarzio, Sébastien Manneville, Lei Sui, \\ Gopi Maguluri, Todd W. Murray, and Ronald A. Roy
}

\begin{abstract}
Acoustophotonic imaging uses ultrasound-modulated scattered light to improve the quality of optical imaging in diffusive media. Experiments that use photorefractive-crystal-based detection have shown that there is a large dc shift in the acoustically modulated or ac optical signal, which could be utilized to further improve optical imaging resolution. We report that photon paths in a diffusive medium were generated by a Monte Carlo simulation, and the optical phase shifts of the various photons induced by the presence of a realistic focused ultrasound beam were calculated. Quantities that characterize the ac and dc signal components were evaluated by use of the calculated phase shifts. It was confirmed that the dc component dominates owing to coherent summation of the contributions from all the photons. (c) 2005 Optical Society of America
\end{abstract}

OCIS codes: $170.3880,110.7050,290.7050,110.7170$.

\section{Introduction}

There has been increasing interest in optically based techniques for biomedical imaging ${ }^{1,2}$ because of the valuable medical information that can be obtained from the optical properties of biological tissue. The primary difficulty is that light in tissue is highly scattered or diffuse, which limits the imaging resolution. ${ }^{3}$ In the early 1990s, new techniques were introduced that combine light with focused ultrasound, offering the possibility of optical imaging at submillimeter resolution limited only by the parameters of the ultrasound field. In these techniques the ultrasound field interacts with diffuse light in the imaging volume by acoustically modulating the phases of photons. The goal is to detect the ultrasound-modulated optical signal, or the acoustophotonic imaging (API)

F. J. Blonigen, A. Nieva, and C. A. DiMarzio (dimarzio@ece.neu. edu) are with the Center for Subsurface Sensing and Imaging Systems, Northeastern University, 360 Huntington Avenue, Boston, Massachusetts 02115. S. Manneville is with the Centre de Recherche Paul Pascal, Avenue Schweitzer, 33600 Pessac, France. L. Sui, G. Maguluri, T. W. Murray, and R. A. Roy are with the Department of Aerospace and Mechanical Engineering, Boston University, 110 Cummington Street, Boston, Massachusetts 02215.

Received 13 August 2004; revised manuscript received 19 January 2005; accepted 29 January 2005.

$0003-6935 / 05 / 183735-12 \$ 15.00 / 0$

(C) 2005 Optical Society of America signal, that contains information about the optical and acoustical properties of the medium in a small region containing the ultrasound focus where the acoustophotonic interaction is strongest. Detection of an ultrasound-modulated signal was first reported by Marks et al., ${ }^{4}$ who, along with Mahan et al., ${ }^{5}$ referred to the acoustophotonic interaction as the ultrasonic tagging of light. Wang et al. ${ }^{6}$ and Wang and Zhao ${ }^{7}$ followed this work by using ultrasound-modulated light to image objects in diffusive media, a technique that they called ultrasound-modulated optical tomography. Others have been developing similar techniques and giving them different names. ${ }^{8-13}$ We use the term API in this paper to refer to any technique that uses ultrasound-modulated light to image in diffusive media. Originally, it referred to a variant technique developed by the present authors that uses an amplitude modulated laser as the optical source. ${ }^{10}$

Recently, theoretical work has been undertaken to explain the process behind the acoustophotonic interaction and to calculate API signal levels., ${ }^{5,14-17}$ Here we are interested in studying the API signals measured by use of a new detection system developed by us that employs a photorefractive crystal (PRC). ${ }^{18}$ Such detection systems enjoy widespread use and have been the subject of many papers. ${ }^{19-21}$ We extended the application of PRC detection systems to API to solve the problem of detecting the API signal in the speckle of diffuse scattered light in tissue. In- 
coherent detection schemes that average out the speckle pattern are precluded and detection of single speckles is frustrated by low light levels. This problem led Boccara and co-workers ${ }^{9}$ to develop a detection system that uses a CCD camera with pixels that are approximately the size of a single speckle to average over the magnitudes of many speckles, leading to a significant increase in the signal-to-noise ratio. Our detection system, however, uses a single-element detector and a PRC to improve API signal strengths. Through nonlinear two-wave mixing of the speckled signal beam and a strong reference beam, the PRC coherently amplifies the speckle pattern through optical means before electronic detection and amplification with the optical detector. Because the PRC can adapt to slow changes in the speckle pattern, the detection scheme to some extent circumvents the problem of speckle decorrelation during measurement in the multispeckle detection technique of Boccara. Through PRC-based detection, we are also able to avoid the problems of incoherent detection with single-element detectors and the low signal-to-noise ratios of pulsed-ultrasound API.

In addition to increasing signal levels, the PRC detection system affects the structure of the API signal, leading to a new feature, a dc component, that had not been studied in work on API until now. The weak API signals that were previously observed consisted principally of a first harmonic or ac component at the acoustic frequency. However, with the PRCbased detection system, a shift occurs in the dc level of the ac component relative to the background dc level that is observed when ultrasound is absent. Because its contributions add coherently, the dc shift actually dominates the first-harmonic component, even though it is second order in the acoustically induced optical phase shifts. The result is that the API signal basically consists of a demodulation of the ultrasound signal, which resembles the envelope function of the original ultrasound pulse as a function of time. In this case the dc component can be used in place of the ac component as a basis for new approaches to API that use pulsed ultrasound waves.

In this study we developed a computational method for calculating the acoustically induced optical phase shifts of photons that follow paths that are typical in an API system, which henceforth will be referred to simply as the phase shifts. It is necessary to have a good sense of their magnitude, as they must not be too small if the dc shift is to be explained as an effect that is second order in the phase shifts. The calculated phase shifts are then used to evaluate quantities that are characteristic of the ac and dc signal components of the API signal detected by use of a PRC-based system. These calculations were performed with a Monte Carlo code that generates random photon paths in a diffusive medium and a finitedifference time-domain simulation of a realistic focused ultrasound beam that, to our knowledge, had not previously been attempted. The emphasis on the explicit calculation of phase shifts makes this study different from previous ones that considered instead the modulation depth of the API signals. ${ }^{14,22}$ An advantage in calculating phase shifts as opposed to the API signal itself is that we are able to consider a wide range of paths that may be significant for API, not just the paths that reach a single detection point, and this helps to reduce computing time and corresponds better to situations in which a lens is used to detect photons from a large area.

In Section 2 of this paper we derive expressions for the ac and dc components of the API signal detected by use of a PRC-based detection system in API as well as expressions for contributions to the phase shifts. In Section 3 we discuss computational modeling of the ultrasound in API and calculation of the phase shifts, while in Section 4 we discuss the optical modeling. In Section 5 we present the results of this study and in Section 6 provide a summary of the paper.

\section{Theoretical Model for the API Signal That Uses a PRC Detection System}

\section{A. Scattered Electric Field and the Acoustically Induced Phase Shifts}

In our modeling of an API system it is assumed that a beam of coherent light is incident onto a diffusive medium composed of a random arrangement of optically scattering particles. Each of the photons of the incident beam executes its own random walk among the particles until it is either absorbed by one of the particles or scattered out of the medium. The scattered electric field incident upon the detection system is denoted $E_{S}$. It may be treated as a superposition of the electric field contributions of the detected photons: $E_{S}=\sum_{j} E_{S j}$. Here $E_{S j}$ is the field that corresponds to the $j$ th photon and its path. The field quantities correspond to one polarization component of the electric field vector that is singled out for detection.

In API, an ultrasound field is imposed on the diffusive medium. Its effect on the scattered photons is to shift the phases of their corresponding fields by modulating them at the acoustic frequency. The field contribution of the $j$ th photon path can be written as $E_{S j}=E_{j} \exp \left(i \Phi_{j}\right)$, where $E_{S j}$ is the field contribution when the ultrasound is absent and

$$
\Phi_{j}=\sum_{m=1}^{N_{j}} \phi_{j m}
$$

is the acoustically induced phase shift for the $j$ th path. It is the sum of the phase-shift contributions $\phi_{j m}$ from each of the $N_{j}$ scattering events of the $j$ th photon located at one of the particles in the $j$ th path.

Two physical mechanisms were considered for the phase-shift contributions: (1) The scattering particles can be displaced by the ultrasound field. This results in Doppler-shifted electric fields at the optical detector. (2) The pressure field can modulate the refractive index along the optical path, thereby modulating the phase of the optical field. This is the Raman-Nath effect. ${ }^{23}$ 
Mechanism (1) was modeled by Leutz and Maret ${ }^{24}$ in a study of ultrasound-modulated light in a diffusive medium. When a particle is displaced from its equilibrium position, the path length changes for a photon that scatters off that particle and results in a phase shift for the photon. To first order in the particle displacements, the phase-shift contribution that is due to mechanism (1) and corresponds to the $m$ th scattering event in the $j$ th path is

$$
\phi_{j m}{ }^{(d)}=\Delta \mathbf{k}_{j m} \cdot \mathbf{x}_{j m}(t),
$$

where $\Delta \mathbf{k}_{j m}$ is the difference between the incoming and outgoing photon wave vectors at the $m$ th particle and $\mathbf{x}_{j m}(t)$ is the displacement of the $m$ th particle from its equilibrium position.

The contributions that are due to mechanism (2) and correspond to the $m$ th scattering event are given by the equation

$$
\phi_{j m}{ }^{(n)}=\int_{\mathbf{r}_{j, m-1}}^{\mathbf{r}_{j m}} k \Delta n(\mathbf{r}, t)\left(\hat{l}_{j m} \cdot d \mathbf{r}\right),
$$

where $\Delta n$ is a small change in the refractive index induced by the ultrasound field and $k$ is the optical wave number. The integral of Eq. (3) gives the phase shift that the $j$ th photon acquires in traversing the straight-line path from particle $m-1$ to the $m$ th particle, located at positions $\mathbf{r}_{j, m-1}$ and $\mathbf{r}_{j m}$, respectively, along the $j$ th path. In Eq. (3), $\hat{l}_{j m}=\mathbf{l}_{j m} /\left|\mathbf{l}_{j m}\right|$, where $\mathbf{l}_{j m}=\mathbf{r}_{j m}-\mathbf{r}_{j, m-1}$ is the vector connecting particle $m-1$ to particle $m$.

The total phase-shift contribution from the $m$ th scattering event is

$$
\phi_{j m}=\phi_{j m}{ }^{(d)}+\phi_{j m}{ }^{(n)} .
$$

The phase shift for the entire $j$ th photon path, $\Phi_{j}$, is the sum of such phase-shift contributions per Eq. (1).

We note that particle displacements $\mathbf{x}_{j m}(t)$ and refractive-index changes $\Delta n$ are time-dependent quantities. They are proportional to the acoustic pressure of the ultrasound field, $p$, which oscillates at acoustic frequency $f_{a}$. Therefore the phase shifts may be written as

$$
\Phi_{j}=-\left|\Phi_{j}\right| \sin \left(\omega_{a} t-\delta_{j}\right),
$$

where $\omega_{a}=2 \pi f_{a}$. Because it is convenient to treat the pressure as the real part of a complex number, the same will be done for the phase shifts: $\Phi_{j}=$ $\operatorname{Re}\left\{-i\left|\Phi_{j}\right| \exp \left[-i\left(\omega_{a} t-\delta_{j}\right)\right]\right\}$. Thus $\left|\Phi_{j}\right|$ is the absolute value of $\Phi_{j}$ when it is expressed in terms of a complex exponential.

\section{B. API Signal}

Given the scattered field $E_{S}$, one may calculate the optical intensity at a detector after the scattered light has passed through a PRC detection system. Inside the PRC, the scattered field is mixed with a strong reference beam of the same optical frequency that was generated by splitting off some light from the laser source that produces the incident beam. Through the photorefractive effect, energy is transferred from the reference beam to the scattered beam, resulting in the coherent amplification of the speckle pattern of the scattered beam. The PRC can adapt to changes in the speckle pattern on the time scale of the PRC's response time, providing one advantage to the use of the PRC detection system. Yet the response time of the PRC is slow enough that it is unresponsive to the high-frequency modulation produced by the ultrasound source, resulting in a relative phase shift between the acoustical and the purely optical contributions to the scattered field that can be used for heterodyning. We write the gain of the PRC as $\exp (\gamma L)$, where $\gamma$ is the gain coefficient and $L$ is the length of the PRC traversed by the scattered light. In general, the gain coefficient may be complex, so we can write $\gamma=\gamma_{R}+\gamma_{1}$, where $\gamma_{R}$ and $\gamma_{I}$ are the real and the imaginary parts, respectively, of the gain coefficient. In addition, there is also absorption of optical fields in the PRC. The optical energy absorption coefficient will be denoted $\alpha$.

The PRC detection system is suited for the detection of transient signals ${ }^{25}$ and thus is suited for the use of pulsed ultrasound in the application of API. In this case, Eq. (5) is only an approximate expression for the phase shifts during the time that the ultrasound pulse interacts with their corresponding photon paths. For ultrasound pulses of several cycles at megahertz frequencies, the pulse duration is short compared with the typical time response of a PRC (longer than a millisecond), such that the PRC will not respond to acoustically induced scattering contributions. Writing the scattered field of the $j$ th path as $E_{S j}=E_{j}+\left[E_{j} \exp \left(i \Phi_{j}\right)-E_{j}\right]$ allows us to separate the acoustical from the purely optical contributions to the scattered field of the $j$ th photon, where we identify the term in brackets in this expression with the acoustical contribution. After the scattered field passes through the PRC it becomes $E_{S j} \rightarrow E_{j} \exp (-\alpha L / 2)\left\{\exp (\gamma L)+\left[\exp \left(i \Phi_{j}\right)-1\right]\right\}$. The acoustical term is not amplified.

An optical detector placed after the PRC detects a signal proportional to the magnitude squared of the total field:

$$
\begin{aligned}
\left\langle\left|E_{S}\right|^{2}\right\rangle= & \exp (-\alpha L) \sum_{j}\left|E_{j}\right|^{2}\left(|\exp (\gamma L)|^{2}\right. \\
& \left.+2 \operatorname{Re}\left\{[\exp (\gamma L)-1]^{*}\left[\exp \left(i \Phi_{j}\right)-1\right]\right\}\right),
\end{aligned}
$$

where the angle brackets denote a statistical average over all arrangements of optical scatterers. The cross terms, which account for the speckle, vanish on statistical averaging. However, even though the detector averages out the speckle, we retain API information in the final signal. In Eq. (6) the first term in bold parentheses in the summand is a dc background signal, which is the signal that one obtains when no ultrasound is present. The remaining term contains the 
phase shift, which is identified as the API signal. The API signal term vanishes when the gain coefficient, $\gamma$, goes to zero, demonstrating that the PRC is essential to our detection scheme. Typically, $|\exp (\gamma L)| \gg 1$ and the API signal increases in proportion to $|\exp (\gamma L)|$ as the PRC gain increases. Equation (6) was derived elsewhere in discussions of PRC detection systems. ${ }^{25}$

When the phase shifts are small, the API signal term in Eq. (6) is proportional to the phase shifts that oscillate sinusoidally at the acoustic frequency. In this case the API signal consists of only a firstharmonic term. For arbitrarily large phase shifts the possibility exists that second- and higher-harmonic components will appear in the API signal, as well as an additional contribution to the dc level that was observed in experiments. ${ }^{18}$ One finds the magnitude of the dc shift and the amplitude of the first harmonic during the period of the API signal pulse by calculating the Fourier coefficients of $\exp \left(i \Phi_{j}\right)$, with $\Phi_{j}$ given by Eq. (5). The dc component of the API signal is characterized by a zeroth-order Bessel function, $J_{0}(x)$ :

$$
\begin{aligned}
\left\langle\left|E_{S}\right|^{2}\right\rangle_{\mathrm{dc}}= & 2 \exp \left(\gamma_{R} L-\alpha L\right) \cos \left(\gamma_{I} L\right) \\
& \times \sum_{j}\left|E_{j}\right|^{2}\left[J_{0}\left(\left|\Phi_{j}\right|\right)-1\right] .
\end{aligned}
$$

The first harmonic or ac component of the API signal is expressed in terms of a first-order Bessel function, $J_{1}(x)$ :

$$
\begin{aligned}
\left\langle\left|E_{S}\right|^{2}\right\rangle_{\mathrm{ac}}= & 4 \exp \left(\gamma_{R} L-\alpha L\right) \sin \left(\gamma_{1} L\right) \\
& \times \sum_{j}\left|E_{j}\right|^{2} J_{1}\left(\left|\Phi_{j}\right|\right) \sin \left(\omega_{a} t-\delta_{j}\right) .
\end{aligned}
$$

Other harmonics contribute to the ac signal, but they are not significant. Thus we identify the first harmonic with the ac component. In Eqs. (7) and (8) we have ignored terms that are zero order in the gain factor because we assume that the gain can be made large.

We can define two quantities that characterize the photon contributions to the ac and dc components of the API signal:

$$
\begin{aligned}
& A_{j}=J_{1}\left(\left|\Phi_{j}\right|\right) \exp \left(i \delta_{j}\right), \\
& D_{j}=J_{0}\left(\left|\Phi_{j}\right|\right)-1 .
\end{aligned}
$$

The quantity $A_{j}$, which is first order in the phase shifts when the phase shifts are not too large, characterizes the $j$ th contribution to the ac component of the API signal, and the quantity $D_{j}$, which is second order in the phase shifts, characterizes the $j$ th contribution to the dc shift. Because $D_{j}$ is only a secondorder quantity, one might expect the dc shift to be dominated by the ac signal component. However, as can be seen from Eq. (8), the ac component is a sum of sinusoidal contributions with different phases $\delta_{j}$ that therefore tend to cancel one another, but all the contributions to the dc shift add constructively. Furthermore, the phase shifts are not necessarily small.

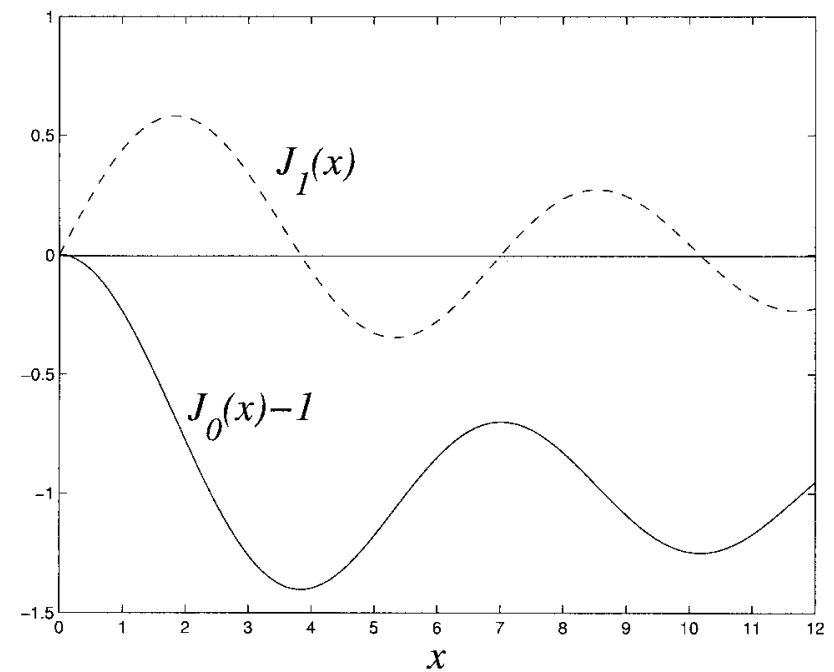

Fig. 1. Plot of $\left[J_{0}(x)-1\right]$ (solid curve) and $J_{1}(x)$ (dashed curve). The function $J_{1}(x)$ characterizes a contribution of a photon to the ac component amplitude of the API signal when the magnitude of the photon's acoustically induced phase shift is substituted for $x$, while the function $\left[J_{0}(x)-1\right]$ characterizes the dc shift. When $x$ is small, $\left[J_{0}(x)-1\right]$ is dominated by $J_{1}(x)$. As $x$ becomes large, $\left[J_{0}(x)-1\right]$ asymptotes to -1 while $J_{1}(x)$ asymptotes to zero, and $\left[J_{0}(x)-1\right]$ dominates in this case.

When the phase shifts are sufficiently large, the factors $\left|D_{j}\right|=\left[J_{0}\left(\left|\Phi_{j}\right|\right)-1\right]$ that contribute to the dc shift become large compared with the quantities $\left|A_{j}\right|=J_{1}\left(\left|\Phi_{j}\right|\right)$ of the first-harmonic terms, as is demonstrated by a plot of the functions $\left[J_{0}(x)-1\right]$ and $J_{1}(x)$ shown in Fig. 1 . As $x$ becomes large, $\left[J_{0}(x)-1\right]$ asymptotes to -1 and $J_{1}(x)$ asymptotes to zero. By large $x$ we mean $\sim 1$ rad or greater. For $x$ greater than $\sim 1 \mathrm{rad}$, the functions $J_{1}(x)$ and $\left[J_{0}(x)-1\right]$ are no longer well described by the lowest nonzero term in their Taylor series expansions near $x=0$, which for these two functions are the linear term and the quadratic term, respectively.

If the dc shift is dominant in the API signal, it may be more effective to use the dc component, instead of the first harmonic, in API. Because it is quadratic in the phase shifts, and thus quadratic in the acoustic pressure, the dc shift is also more sensitive to large spatial pressure variations in the ultrasound beam and could be exploited to enhance imaging resolution.

\section{Numerical Model for the Focused Ultrasound Field and the Calculation of the Phase Shifts}

\section{A. Numerical Simulation of the Ultrasound Field}

The modeling of the ultrasound field and its effects on the motion of particles in an optically diffusive medium has been rather simplistic in work on API. Usually, plane-wave ultrasound fields are considered in the analysis. ${ }^{14-17}$ For the focused ultrasound used in API, the acoustic field can be better modeled as a Gaussian beam. However, the standing-wave Gaussian beam solution for resonator cavities, ${ }^{26}$ although it is familiar, can be only an approximation of the real ultrasound field because it is a traveling wave gen- 
erated by a focusing transducer and therefore does not have reflection symmetry centered about the focus. In addition, it is often assumed that the motion of the particles simply follows the motion of the surrounding fluid or elastic solid. But rheology alters this motion and can lead to noticeable changes in the values for the acoustically induced phase shifts, depending on the mass density of the scattering particles. Moreover, nontrivial acoustical modeling is required for rheological and viscous effects to be taken into account.

For these reasons we decided to employ a more exact model for the ultrasound in API and its effects on the motion of the scattering particles in an optically diffusive medium. The basis for the model is a nonlinear propagation equation for acoustic waves in an absorbing Newtonian fluid called the Westervelt equation ${ }^{27}$ :

$$
\nabla^{2} p-\frac{1}{c_{a}^{2}} \frac{\partial^{2} p}{\partial t^{2}}+\frac{\delta}{c_{a}^{4}} \frac{\partial^{3} p}{\partial t^{3}}+\frac{\beta}{\rho c_{a}^{4}} \frac{\partial^{2} p^{2}}{\partial t^{2}}=0 .
$$

In Eq. (11), $p$ is the acoustic pressure, $c_{a}$ is the speed of sound in the fluid, $\delta$ is the diffusivity of sound, which accounts for the viscous effects, $\rho$ is the density of the fluid, and $\beta$ is the coefficient of nonlinearity. The subscript $a$ is used to indicate acoustical quantities that could be misconstrued as optical quantities.

The Westervelt equation ignores the presence of particles in the fluid. It is assumed that there is negligible scattering of the ultrasound by the particles if most of the acoustic energy can be channeled into a high-pressure focal region, which is evident from ultrasonic experiments in tissue. This assumption is valid for a sufficiently dilute suspension of small particles. In the simulation the particles are micrometer sized and possess weak acoustic contrast. They are small compared with the acoustic wavelength, which is hundreds of micrometers in the megahertz frequency range. Also, the typical average distance between particles is at least an order of magnitude larger than the particle size. Thus the weakly scattered pressure of one particle should have no effect on surrounding particles.

In our study the Westervelt equation was solved numerically by use of a two-dimensional axisymmetric finite-difference time-domain code. ${ }^{28}$ The pressure of an ultrasound field was computed inside a cylindrically shaped, acoustically homogeneous optically diffusive medium of $7 \mathrm{~cm}$ radius and $12 \mathrm{~cm}$ length. The diffusive medium was water with a suspension of randomly placed spherical particles of radii $a$ $=0.4 \mu \mathrm{m}$. The diffusive parameters are given in Section 4 below. The ultrasound field in the simulation was generated by a circular focusing transducer with an aperture $D=7 \mathrm{~cm}$ in diameter and a focal length $d=6.26 \mathrm{~cm}$. The center of the transducer was coincident with the center of one end of the cylindrical computational domain. The transducer was assumed to operate at constant frequency after turn-on, with

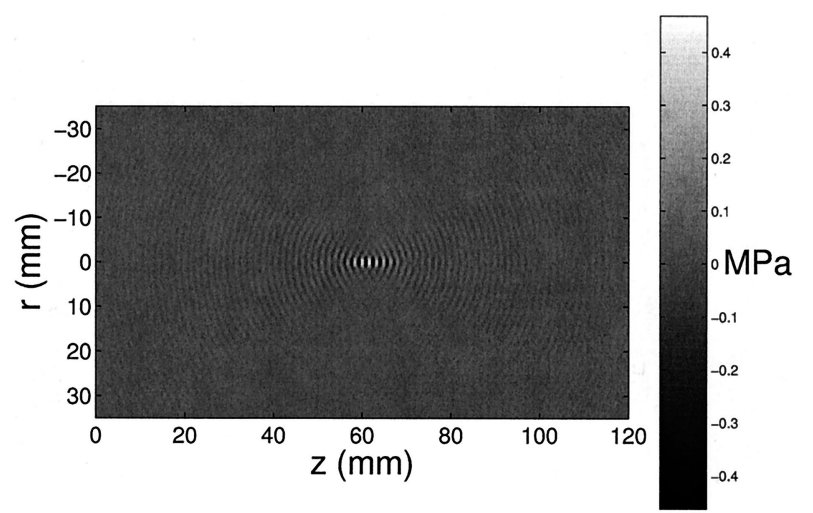

Fig. 2. Intensity plot of the pressure in water generated by a circular focusing transducer with an aperture of $7 \mathrm{~cm}$ diameter and a focal length of $6.26 \mathrm{~cm}$ operating at $1 \mathrm{MHz}$. The pressure was computed with a finite-difference time-domain code to solve the Westervelt equation [Eq. (11)]. The center of the transducer is at $r=z=0$. There is a high-pressure focal region along the axis near $z=6 \mathrm{~cm}$.

its frequency $f_{a}$ set to $1 \mathrm{MHz}$. The pressure amplitude was set to $10^{4} \mathrm{~Pa}$ at the surface of the transducer. Equation (11) was solved in cylindrical coordinates $(r, z)$ on a grid with $\delta_{r}=\delta_{z}=0.1 \mathrm{~mm} \approx \lambda_{a} / 15$, where $\lambda_{a}=c_{a} / f_{a}$, and time step $\delta t=1 /\left(100 f_{a}\right)$. The simulation was run for a time long enough to reach a steady-state solution over the entire computational domain. Thus we simulated a continuous-wave ultrasound beam, even though ultrasound pulses were used in the experiments of Ref. 18. This was simpler and about as accurate as simulating a pulsed ultrasound beam of several cycles. It also facilitates the calculation of the sinusoidal phase shifts [Eq. (5)].

The pressure field is a function of space and time: $p=p(\mathbf{r}, t)$. In Fig. 2, the pressure at time $t=$ $100 \mu \mathrm{s}$ (after $10^{5}$ time steps) is given by a twodimensional gray-scale plot as a function of spatial variables $r$ and $z$. The origin of the coordinate system is located at the center of the transducer. Figure 2 shows that the ultrasound field has a roughly Gaussian beam shape and that there is a high-intensity focus at one focal length from the center of the transducer. We define the focal region of the ultrasound beam as that region where the pressure amplitude is no less than $3 \mathrm{~dB}$ down from the maximum pressure in the beam. For this simulated pressure field, the focal region has roughly the shape of an ellipsoid that is $\sim 1 \mathrm{~cm}$ long parallel to the ultrasound beam axis and close to $2 \mathrm{~mm}$ wide in the focal plane. The wavelength of the propagating sound is $\sim 1.5 \mathrm{~mm}$, which is what we expect for a frequency of $f_{a}=1 \mathrm{MHz}$. The maximum pressure obtained in this numerical simulation was $469 \mathrm{kPa}$ at the focus. This is about what we should expect for a source pressure of $10^{4} \mathrm{~Pa}$ and a gain of $G=2 \pi f_{a} D^{2} / 8 c_{a} d \approx 42$ for the transducer.

At every point on the grid, we specified a complex value for the pressure field. Assuming a steady-state pressure, two sets of pressure values over the grid of the computational domain separated in time by a quarter of an acoustic cycle can be used to determine 
the complex value of the pressure:

$$
A(\mathbf{r})=p\left(\mathbf{r}, t_{o}\right)+i p\left(\mathbf{r}, t_{o}+\frac{1}{4 f_{a}}\right) .
$$

Here, $t_{o}=100 \mu \mathrm{s}$. The pressure magnitude at time $t_{o}$ is given by $|A(\mathbf{r})|$. The complex phase of $A(\mathbf{r})$ includes the term $-\omega_{a} t_{0}$. We also specified a local acoustic wave vector at every point on the grid, defined as

$$
\mathbf{k}_{a}(\mathbf{r})=\varepsilon \frac{\nabla A(\mathbf{r})}{i A(\mathbf{r})},
$$

where $\varepsilon= \pm 1$ is chosen such that $\mathbf{k}_{a}$ always points in the direction of propagation.

\section{B. Acoustically Induced Motion of Scattering Particles}

The presence of an acoustic pressure field leads to motion of the optical scatterers in the diffusive medium, which in turn shifts the phases of the photon fields through mechanism (1). The displacement, $\mathbf{x}$, of a scattering particle from its equilibrium position is determined from Newton's second law:

$$
m \frac{\partial^{2} \mathbf{x}}{\partial t^{2}}=-\iint_{S} p \mathbf{n} \mathrm{d} s-6 \pi \rho \nu a\left(\frac{\partial \mathbf{x}}{\partial t}-\mathbf{y}\right) .
$$

The left-hand side of Eq. (14) is the mass of the particle, $m$, multiplied by its acceleration. The righthand side is the expression for the force on the particle. The first term on the right-hand side is the direct radiation stress that is due to the pressure field. It is an integral of the pressure over surface $S$ of the particle, where $\mathbf{n}$ is the outward normal to surface $S$. The second term is the Stokes expression for the viscous drag force on the particle, where $v$ is the fluid kinematic viscosity $\left(9 \times 10^{-7} \mathrm{~m}^{2} \mathrm{~s}\right.$ for water $)$ and $\mathbf{v}$ is the local acoustically induced fluid velocity. The viscous boundary layer thickness, $\sqrt{2 v / \omega_{a}}$, is comparable to $a$, so viscous effects cannot be neglected. From the usual linear relationship between acoustic velocity and acoustic pressure, we find that $\mathbf{v}=A \mathbf{k}_{\alpha} / \rho \omega_{\alpha}$.

A discussion of the evaluation of the surface integral in Eq. (14) is in order. It is useful to write the pressure on the particle's surface as $p=p_{i}+p_{s}$, where $p_{i}$ is the local pressure field incident upon the scatterer and $p_{s}$ is the pressure scattered off the particle's surface. Because the scatterers are small compared with the acoustic wavelength, we can approximate the local pressure field incident upon a particle by a plane wave: $p_{i}=A\left(\mathbf{r}_{o}\right) \exp \left[i k_{a}\left(\mathbf{r}_{o}\right) \cdot(\mathbf{r}\right.$ $\left.\left.-\mathbf{r}_{o}\right)-i \omega_{a}\left(t-t_{o}\right)\right]$. Here $\mathbf{r}_{o}$ is taken to be the position of the center of the spherical particle at equilibrium. In the numerical simulation the value for $\mathbf{r}_{o}$ was rounded to the nearest point on the grid. In the evaluation of the surface integral, surface $S$ is taken to be the particle surface at equilibrium. The displacement of the particle is assumed to be small compared with the particle's radius, so $S$ is always approximately the surface of the particle when it is in motion. This assumption is borne out by the numerical simulation and thus constitutes an $a$ posteriori check of the assumption.

Employing the assumption that $a$, the particle radius, is small compared with the acoustic wavelength, one finds that

$$
-\iint_{S} p_{i} \mathbf{n} \mathrm{d} s=-i V A\left(\mathbf{r}_{o}\right) \mathbf{k}_{a}\left(\mathbf{r}_{o}\right) \exp \left[-i \omega_{a}\left(t-t_{o}\right)\right],
$$

where $V=(4 / 3) \pi a^{3}$ is the volume of the particle.

Yosioka and Kawasima ${ }^{29}$ provide an expression for the weakly scattered pressure that is due to a plane wave incident upon a sphere. The spherical Bessel and Hankel functions of the expression can be expanded in powers of $k_{a} a$, where $k_{a}=\left|\mathbf{k}_{a}\right| \approx \omega_{a} / c_{a}$ is the acoustic wave number. Because the acoustic wavelength is much larger than the radius of the particle, $k_{a} a$ is a small quantity. For this reason the expansions were truncated at the lowest-order term in $k_{a} a$. The resultant integration of $p_{s}$ is then

$$
\begin{aligned}
-\iint_{S} p_{s} \mathbf{n} \mathrm{d} s= & -i \frac{\rho^{\prime}-\rho}{2 \rho^{\prime}+\rho} V A\left(\mathbf{r}_{o}\right) \mathbf{k}_{a}\left(\mathbf{r}_{o}\right) \\
& \times \exp \left[-i \omega_{a}\left(t-t_{o}\right)\right]
\end{aligned}
$$

where $\rho^{\prime}=m / V$ is the material density of the particle.

We seek solutions to Eq. (14) that are time harmonic at the acoustic frequency:

$$
\mathbf{x}(t)=i \chi \frac{A\left(\mathbf{r}_{o}\right) \mathbf{k}_{a}\left(\mathbf{r}_{o}\right)}{\rho \omega_{a}{ }^{2}} \exp \left[-i \omega_{a}\left(t-t_{o}\right)\right] .
$$

Here $\chi$ is a constant complex number. For $\chi=1, \mathbf{x}$ is identical to the displacement of the fluid at $\mathbf{r}_{o}$ that would obtain if the particle were not there. Substituting Eqs. (15)-(17) into Eq. (14) and solving for $\chi$, we find that

$$
\chi=\left(1-\frac{3 \rho}{2 \rho^{\prime}+\rho} i \omega_{a} \tau\right) /\left(1-i \omega_{a} \tau\right),
$$

where $\tau=2 \rho^{\prime} a^{2} / 9 \rho v$ is a viscous characteristic time. Depending on $\rho$ and $\rho^{\prime}, \chi$ may be significantly different from 1. For biological scatterers in tissue it is likely that $\chi \approx 1$, in which case the particle tracks the fluid motion as a Lagrangian tracer.

The time-harmonic solutions for the displacements imply that the particles oscillate sinusoidally about their equilibrium positions under the influence of the ultrasound field. This solution does not account for progressive drift of the particles away from their equilibrium positions owing to acoustic streaming. Acoustic streaming is a higher-order acoustical effect 
that can be neglected to a first approximation but may have important consequences for API. For example, there is no acoustic streaming in tissue, but there could be in blood. There should be no acoustic streaming in tissue-mimicking gel phantoms, however. It should also be noted that we did not model the effects of Brownian motion on the displacement of the particles, as was done in the analyses of others. ${ }^{13,16,17}$ The particles are assumed to be fixed at their equilibrium positions before the ultrasound is turned on.

\section{Calculation of Phase-Shift Contributions}

Using computed values for the particle displacements, one can calculate the phase-shift contributions that are due to mechanism (1) by way of Eq. (2). In Eq. (2), the quantities $\mathbf{x}_{j m}$ are the particle displacements for $\mathbf{r}_{o}=\mathbf{r}_{j m}$, where $\mathbf{r}_{j m}$ is the position of the $m$ th particle in the $j$ th photon path. For definiteness, the time is set to $t_{o}$.

There are also the contributions that are due to the acoustically induced optical refractive-index changes in the fluid given by Eq. (3). To first order, the acoustically induced refractive-index change, $\Delta n$, is linearly related to the acoustic pressure through the equation $\Delta n=\eta p$, where proportionality constant $\eta$ is called the piezo-optical coefficient (1.466 $\times 10^{-10} 1 / \mathrm{Pa}$ in water). The integral of Eq. (3) is over the straight line joining particles $m-1$ and $m$ along the $j$ th photon path. Rather than numerically evaluating the integral by using the computed values for $p$, we assumed that the local pressure along the path of integration is a plane wave. This approximation should hold if the mean free photon path between scattering events is not too long. For biological tissue, the mean free photon path is typically shorter than an acoustic wavelength in the low-megahertz frequency range. ${ }^{30,31}$

Using the expression $A\left(\mathbf{r}_{j m}\right) \exp \left[i \mathbf{k}_{a}\left(\mathbf{r}_{j m}\right) \cdot\left(\mathbf{r}-\mathbf{r}_{j m}\right)\right.$ $\left.-i \omega_{a}\left(t-t_{o}\right)\right]$ for the local plane wave and setting $t$ $=t_{o}$ yields for Eq. (3)

$$
\phi_{j m}{ }^{(n)}=k \eta \int_{\mathbf{r}_{j, m-1}}^{\mathbf{r}_{j m}} A\left(\mathbf{r}_{j m}\right) \exp \left[i \mathbf{k}_{a}\left(\mathbf{r}_{j m}\right)\left(\mathbf{r}-\mathbf{r}_{j m}\right)\left(\hat{l}_{\mathrm{jm}} \mathrm{d} \mathbf{r}\right) .\right.
$$

Evaluating the integral of Eq. (19) yields

$$
\phi_{j m}{ }^{(n)}=-i k \eta A\left(\mathbf{r}_{j m}\right)\left|\mathbf{1}_{j m}\right| \frac{\left[1-\exp \left(-i \theta_{i m}\right)\right]}{\theta_{j m}}
$$

for the phase-shift contributions that are due to refractive-index changes, where $\theta_{j m}=\mathbf{k}_{a} \cdot \mathbf{r}_{j m} \cdot \mathbf{l}_{j m}$.

With the calculated phase-shift contributions that are due to mechanisms (1) and (2) in hand, the phase shifts, $\Phi_{j}$, may be calculated from Eqs. (1) and (4). Inasmuch as the computed pressure and particle displacements are complex and time harmonic at the acoustic frequency, so are the calculated phase shifts. Therefore they may be expressed in the form
$-i\left|\Phi_{j}\right| \exp \left[-i\left(\omega_{a} t_{o}-\delta_{j}\right)\right]$, where one finds the real, physical phase shifts by taking the real part, which yields Eq. (5). They correspond to the phase shifts at a time $t_{o}=100 \mu \mathrm{s}$ after the focusing transducer is turned on. In API, light travels from the source to the detector in nanoseconds, which is a very short time compared to an acoustic cycle, so we can assume that the optical phase shifts depend on instantaneous values of the acoustical quantities at a given moment in time.

\section{Optical Modeling of Light Propagation in the Diffusive Medium}

A Monte Carlo computer code was used to model the propagation of photons through the optically diffusive medium of an API setup. It is a modification of a code developed by Prahl et al. ${ }^{32}$ that was used in some of our previous research ${ }^{22}$ and generates the random paths taken by photons as determined by the parameters of the diffusive medium, i.e., scattering coefficient $\mu_{s}$, absorption coefficient $\mu_{a}$, and anisotropy $g$. The code assumes a Henyey-Greenstein ${ }^{33}$ phase function, which can be expressed solely in terms of the anisotropy.

With this code we simulated the diffusion of photons through the cylindrically shaped, optically diffusive, and acoustically homogenous medium described in Section 3 in which a focused pressure field is imposed. The diffusive medium was assigned a scattering coefficient of $\mu_{s}=100 \mathrm{~cm}^{-1}$, which is of the same order of magnitude as that of biological tissues. ${ }^{30}$ The anisotropy was set to 0.9 , and the absorption coefficient was set to $1 \mathrm{~cm}^{-1}$. The scattering particles were taken to be polystyrene spheres of radius $0.4 \mu \mathrm{m}$ (for polystyrene $\chi=1$ ). Besides the particles, the diffusive medium was assumed to be filled with water. The optical wavelength in water was set at $397 \mathrm{~nm}$ for this simulation, which corresponds to the $532 \mathrm{~nm}$ wavelength in air of the green doubled Nd:YAG laser used in the experiments of Ref. 18.

In the simulation, photons were launched into the diffusive medium at a point $1 \mathrm{~cm}$ away from the ultrasound axis in the focal plane of the ultrasound beam. The photons were launched toward the ultrasound focus.

As explained in Section 1, there was not just one detection point of interest but a region of points, which we refer to as the detection region. Figure 3 shows the domain of this region as seen in the plane perpendicular to the acoustic axis, which is the $x-y$ plane for the coordinate system indicated in the figure. The detection region appears to be in the shape of a half-annulus, with an inner radius of $0.75 \mathrm{~cm}$ and an outer radius of $1.25 \mathrm{~cm}$ that are both centered about the acoustic axis, the origin of the $x-y$ plane. The half of the annulus where $y>0$ constitutes the detection region. The launch point, located at $x$ $=0 \mathrm{~cm}$ and $y=-1 \mathrm{~cm}$, is in the region $y<0$. All the points in the detection region are at least a centimeter away from the launch point, which is typical of API configurations. Figure 3 shows only the cross- 


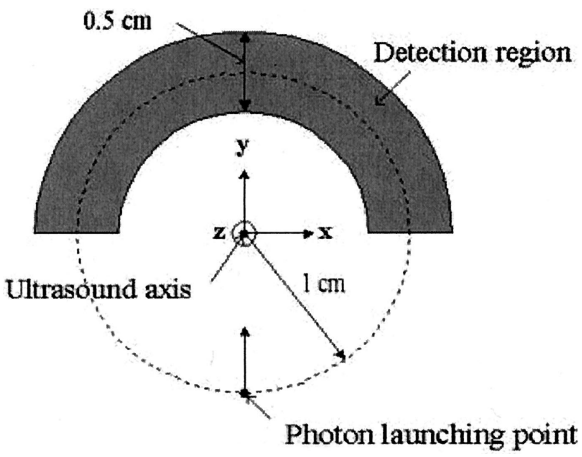

Fig. 3. View down the ultrasound axis, indicating the setup for the optical modeling of the API system as described in Section 4. For the coordinate system indicated, this figure shows the $x-y$ plane with the origin at the ultrasound axis. All photons are launched toward the focus from $1 \mathrm{~cm}$ away, with the launch direction along the $y$ axis. The detection region has a cross-sectional shape of a half-annulus of $0.5 \mathrm{~cm}$ in thickness. The detection region extends out of the page for $12 \mathrm{~cm}$ parallel to the ultrasound beam.

sectional area of the detection region. The region is actually three dimensional and extends the length of the cylindrical domain of the diffusive medium.

We were also interested in distinguishing photons that traveled through different regions of the diffusive medium. In particular, we were interested in distinguishing between those photons that traveled through or close to the ultrasound focus from those that did not. To this end, four hollowed cylindrical regions were considered. Their centers were coincident with one another and the ultrasound focal point. Figure 4 shows a side view of the cylindrical regions with their perimeters outlined in black, overlaid upon a two-dimensional gray-scale plot of the pressure magnitude, $|A(\mathbf{r})|$. The regions are labeled numerically, from 1 to 4 , in order of size from smallest to largest. Region 1 is $2 \mathrm{~mm}$ in diameter and $6 \mathrm{~mm}$ in

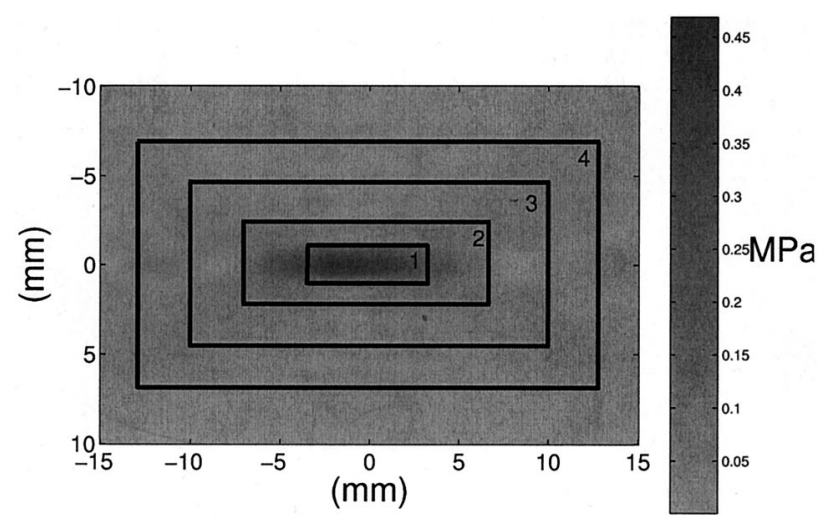

Fig. 4. Side view of four hollowed cylindrical regions of interest in the diffusive medium, with their perimeters shown in black. They are superimposed upon an intensity plot of the pressure magnitude of the numerically calculated ultrasound beam. The horizontal axis is the acoustic axis. The centers of all four hollowed cylinders are at the ultrasound focus, which is at coordinates $(0,0)$ in this plot. Region 1 is not hollowed and is almost entirely contained in the ultrasound focus. Other regions do not contain the smaller regions and thus tend to lie outside the focal region. length, is not hollowed, and is located almost wholly within the ellipsoidally shaped ultrasonic focal region. Region 2 is $4 \mathrm{~mm}$ in diameter and $1.4 \mathrm{~cm}$ in length, and its bounding outer surface contains the entire focal region. Region 3 is $8 \mathrm{~mm}$ in diameter and $2 \mathrm{~cm}$ in length. Region 4 is $12 \mathrm{~mm}$ in diameter and $2.6 \mathrm{~cm}$ in length. Each of regions $1-3$ is part of the hollowed-out volume of the next-larger hollowed cylinder and thus is not considered part of any of the larger regions. Each photon path generated by the Monte Carlo code is labeled by the number of the smallest region through which it passed.

The Monte Carlo simulation launched 200,000 photons from the launch point shown in Fig. 3 and propagated each photon 500 steps. With the absorption coefficient set low, photons are able to propagate a few centimeters before being absorbed. In the simulation, most of the photons that reached the detector region did so within $\sim 400$ steps, beyond which point a photon has less than a $1 \%$ chance of not having been absorbed. In all, 3603 photons reached the detector region. A photon was considered detected once it had entered the detector region and remained there for 10 steps, at which point the photon path was truncated and the rest of the steps in the path generated by the Monte Carlo code, up to the 500th step, were ignored. It is this set of 3603 truncated paths of the original 200,000 that was detected and formed the set of photon paths that we analyzed.

The phase shifts of the detected photons were then calculated according to the prescriptions set forth in Sections 2 and 3 . The calculations of both the photon paths and their corresponding phase shifts were done with the Matlab 6.0 applications program. The total computation time for both calculations was $\sim 90 \mathrm{~min}$ on a standard personal computer.

\section{Computational Results}

Figure 5 shows the results of the phase-shift calculations for the 3603 detected photons in the optical simulation. Recall that the calculated phase shifts are complex and assumed to be of the form $\Phi_{j}=$ $-i\left|\Phi_{j}\right| \exp \left[-i\left(\omega_{a} t_{o}-\delta_{j}\right)\right]$. The histograms in Figs. 5 (a) and 5(b) give the distribution of the magnitudes and arguments of the complex phase shifts of the detected photons, respectively. The values for both the phase shifts and their arguments are given in degrees.

For the magnitude distribution in Fig. 5(a), with histogram bins spaced by $2^{\circ}$, the most populated bin is the phase-shift interval $2^{\circ}-4^{\circ}$, which includes nearly 700 detected photons. The average magnitude of the phase shifts, $\overline{\left|\Phi_{j}\right|}$, is $12.2^{\circ}$, with a standard deviation of $\sim 15.2^{\circ}$. (In this section, averages over detected photon quantities are represented by overbars.) The phase shifts of a number of photons have much higher magnitudes than the average, with one magnitude nearly $200^{\circ}$. These correspond to photons that have passed near the ultrasound focus. Even among these photons, however, the magnitudes of their phase shifts vary considerably. This wide dif- 

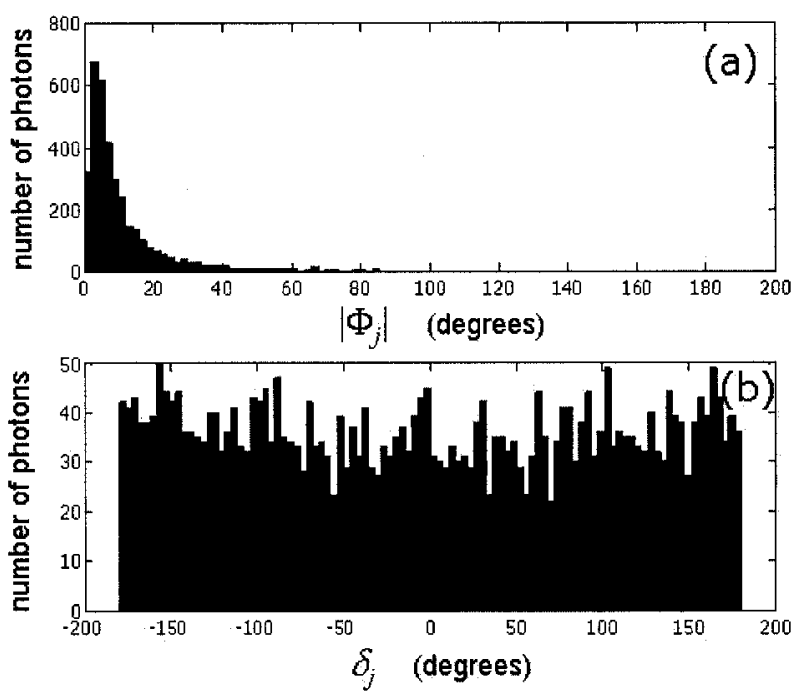

Fig. 5. Distribution of the complex phase shifts, $\Phi_{j}$, for the 3603 detected photons in the simulation of the API system by use of the numerical model for the ultrasound as described in Section 3. (a) The magnitude of the phase shifts. The histogram bins have a width of $2^{\circ}$. The average magnitude is $12.2^{\circ}$. The standard deviation is $15.2^{\circ}$. (b) The arguments of the phase shifts to within a global phase factor. The average is $-5.4^{\circ}$ and the standard deviation is $107^{\circ}$. There are 100 histogram bins equally spaced from $+180^{\circ}$ to $-180^{\circ}$.

ference results from the fortuitous combination of complex contributions to the phase shifts from different scattering sites along the photon paths, yielding large phase shifts for some photons but not for others.

Figure 5(b) gives the histogram for $\delta_{j}$, the arguments of the complex phase shifts after global phase $-\left(\omega_{a} t_{o}+\pi / 2\right)$ is omitted. The average argument is $-5.4^{\circ}$ with a large standard deviation of $107^{\circ}$. It clearly shows that the arguments of the phase shifts are spread over all angles $-180^{\circ} \leq \delta_{j} \leq 180^{\circ}$ in the complex plane. This result is to be expected from the fact that contributions to the phase shift from each scattering event, $\phi_{i m}$, are proportional to the local complex pressure whose argument passes through $2 \pi$ with each acoustic cycle.

These results were checked against phase-shift calculations that were repeated with an analytical Gaussian beam model for the ultrasound beam as opposed to the numerical model discussed in Section 3. The Gaussian beam model contains one free parameter, ${ }^{26}$ the divergence, which was set to $22^{\circ}$. The maximum pressure magnitude was set to $0.47 \mathrm{MPa}$. These settings were chosen such that the Gaussian beam closely approximated the ultrasound beam of the numerical model. The phase shifts were recalculated from the Gaussian beam model with $\chi$ as defined in Eq. (18) set to zero because the Gaussian beam model does not include viscous effects. A histogram similar to the one shown in Fig. 5 is shown in Fig. 6. The distributions of photon phase shifts for the analytical model of the ultrasound look similar to that for the numerical model. However, there is a much larger number of photons with zero phase shift
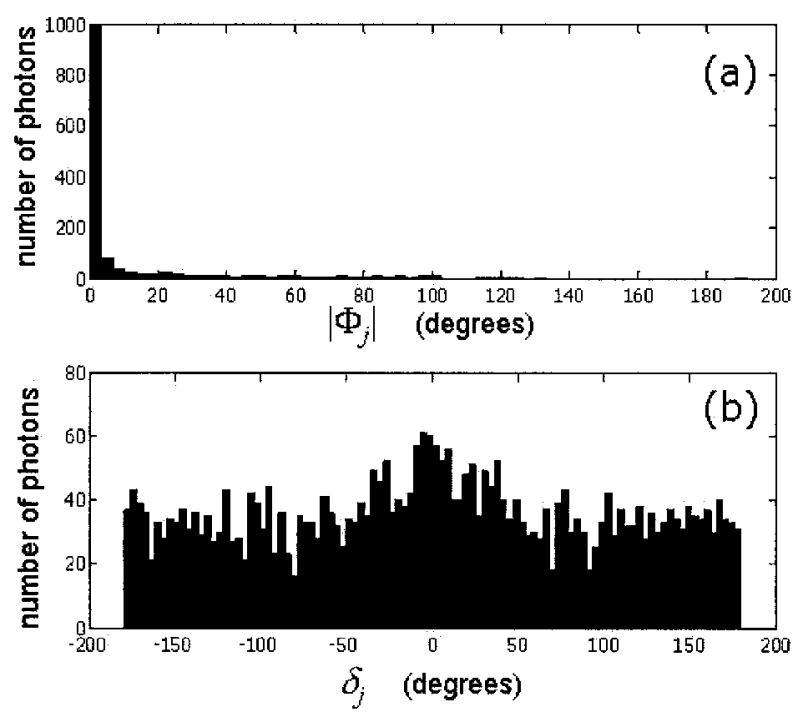

Fig. 6. Distribution of the complex phase shifts, $\Phi_{j}$, for the 3603 detected photons in the simulation of the API system by use of the analytical Gaussian beam model for the ultrasound. (a) The magnitude of the phase shifts. The histogram bins have a width of $\sim 3.5^{\circ}$. The average magnitude is $8.8^{\circ}$. The standard deviation is $31^{\circ}$. (b) The arguments of the phase shifts to within a global phase factor. The average is $0.86^{\circ}$ and the standard deviation is $99.9^{\circ}$. There are 100 histogram bins equally spaced from $+180^{\circ}$ to $-180^{\circ}$.

when the analytical model is used for the ultrasound. The number of photons with zero-phase-shift magnitude is actually larger than the 1000 indicated in Fig. 6(a). In the numerical model the number of photons with zero phase shift is much smaller, which is probably more realistic. The average magnitude of the phase shifts for the analytical model is $8.8^{\circ}$, and the standard deviation is $31^{\circ}$. The average magnitude is somewhat smaller than the average magnitude for the realistic beam $\left(12.2^{\circ}\right)$, but it is approximately the same order of magnitude. The standard deviation is much larger for the Gaussian beam results than for numerical model results. This may be so because there is less leakage of acoustic energy into regions that are far from the ultrasonic beam axis in the simplified case of the Gaussian beam. Thus the photons that missed the focus had small phase shifts.

Returning to the discussion of the results for the numerical model, we found that, though on average the magnitude of the phase shifts of our detected photons was $\sim 12^{\circ}$, there are many other photons that acquire much larger phase shifts depending on how near they come to the ultrasound focus, as one can see from Fig. 5(a). Figure 7 shows the average magnitude of the phase shift for each subset of the total set of detected photons that passed through one of the four hollowed cylindrical regions of interest shown in Fig. 4. In the simulation the photons were labeled 1-4 according to the innermost region that the photon reached along its path. The photons were then grouped into four subsets according to those labels. Photons that passed through region 1 passed the closest to the focal point and hence acquired the largest phase shifts. The average magnitude of the phase 


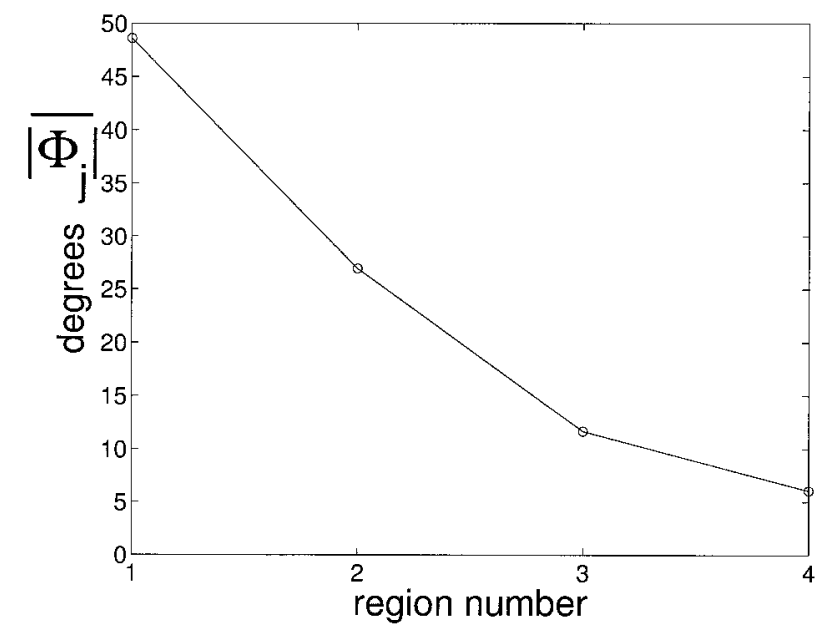

Fig. 7. Average phase-shift magnitudes of four subsets of the detected photons grouped according to the innermost region through which they passed; the regions are those indicated in Fig. 4. These magnitudes were calculated with the numerical model for the ultrasound. Photons have larger phase shifts the closer they pass by the focus. Thus the largest average phase-shift magnitude is for those photons that passed through region 1 .

shift for these photons was $48.5^{\circ}$. A given photon passing only through region 4 will pass far from the focal point and have a small phase shift. The average magnitude of the phase shifts for these photons was $6.0^{\circ}$.

All the photons that passed through regions 1 and 2 passed through some part of the ellipsoidally shaped focal region. Thus, according to Fig. 7, one should expect a photon that passes through the focal region in the API setup modeled in the simulation to have a phase-shift magnitude of $\sim 30^{\circ}$ or greater. These calculations confirm the hypothesis that the phase shifts are large for photons that pass sufficiently near the ultrasound focus (recall from Section 2 that by "large" we mean $\sim 1 \mathrm{rad}$ or greater). It should be emphasized that it is not necessary to go to unreasonably high pressures to generate these large phase shifts. The phase shifts will probably be even larger for a typical API system similar to the one modeled in this simulation. The peak pressure in our simulation was $469 \mathrm{kPa}$. For pulsed ultrasound it is possible to work as high as $1-3 \mathrm{MPa}$ in clinical applications, depending on the frequency; and higher pressures will yield larger phase shifts. However, it is desirable to work even in the infrared range in optical tissue imaging, and longer optical wavelengths will decrease the phase shifts.

Because of these large phase shifts, it is inappropriate to ignore all API effects that are second order in the phase shifts, such as the dc shift effect in the API signal. Let us consider the quantities $A_{j}$ and $D_{j}$, defined by Eqs. (9) and (10), which characterize, respectively, the ac and dc contributions to the API signal from the $j$ th photon. The absolute values of $A_{j}$ and $D_{j}$ evaluated at the average magnitude for the phase shift give an idea of the sizes of these quantities. We find $\left|A_{j}\right|=0.106$ and $\left|D_{j}\right|=0.011$ when they are evaluated at $\overline{\left|\Phi_{j}\right|}$. The quantity $\left|D_{j}\right|$ is $\sim 10$ times smaller than $\left|A_{j}\right|$ at the average phase-shift magnitude. However, $A_{j}$ and $D_{j}$ characterize only contributions to the API signal from the $j$ th photon. The sizes of the ac and dc components are characterized by the absolute values of these factors averaged over all photon contributions in the simulation. For the AC component we obtain $\left|\bar{A}_{j}\right|$; for the dc component we obtain $\left|\bar{D}_{j}\right|$. In this case $\left|\bar{D}_{j}\right|$ is much larger than $\left|\bar{A}_{j}\right|$. This is so because the ac contributions, $A_{j}$, are complex, and each contribution has its own phase. When they are averaged, the ac contributions tend to cancel one another, while the dc contributions, $D_{j}$, add coherently. Furthermore, we note that the quantity $\left|\bar{D}_{j}\right|$ is larger than the quantity $\left|D_{j}\right|$ evaluated at the average phase-shift magnitude. This is so because the quantity $D_{j}$ is second order in the phase shifts, so the terms that correspond to the largest phase shifts in the averaging of $D_{j}$ tend to increase $\left|\bar{D}_{j}\right|$ beyond the value of $\left|D_{j}\right|$ when they are evaluated at $\overline{\left|\Phi_{j}\right|}$. (Note that the averages $\left|\bar{A}_{j}\right|$ and $\left|\bar{D}_{j}\right|$ are not averages weighted by $\left|E_{j}\right|^{2}$. Such weights are fairly uncorrelated with the values of $A_{j}$ and $D_{j}$, and thus we do not expect them to contribute to our qualitative understanding of the ac and dc components.)

Though it should be noted that $\left|\bar{A}_{j}\right|$ and $\left|\bar{D}_{j}\right|$ are not rigorous predictions of the ac and dc components, our calculations confirm that a dc component in API signals can be dominant when one is using a PRC detection system, which was suggested at the end of Section 2 and was seen in experiments. In addition, as $\left|A_{j}\right|$ asymptotes to 0 and $\left|D_{j}\right|$ asymptotes to -1 as the phase shifts become larger, which can be achieved by use of higher pressures, the dc shift will be even more dominant in some situations than was found in our calculation. In this case it is more advantageous to use the dc shift rather than the ac signal amplitude in API.

What should also prove beneficial to imaging in API is that the dc shift is more effective than the ac signal in discriminating between those photons that travel through the focal region from those photons that do not. We examined the quantities $A_{j}$ and $D_{j}$ for the subsets of detected photons labeled $1-4$. Figure 8 shows plots of $\left|\bar{A}_{j}\right|$ and $\left|\bar{D}_{j}\right|$ averaged over the contributions of photons from each of the four subsets. The solid curve gives the values of $\left|\bar{D}_{j}\right|$; the dashed curve gives the values for $\left|\bar{A}_{j}\right|$. Thus the photons that passed closest to the focal point (had smaller label numbers) had the largest values for $\left|\bar{A}_{j}\right|$ and $\left|\bar{D}_{j}\right|$, as they had the largest phase shifts.

Comparing the solid and dashed curves, we note that the values of $\left|\bar{D}_{j}\right|$ are all larger than those of $\left|\bar{A}_{j}\right|$ because the contributions to the dc component, characterized by $\left|\bar{D}_{j}\right|$, add coherently. The values for $\left|\bar{D}_{j}\right|$ also increase at a higher rate for decreasing region numbers, which correspond to photon sets that are increasingly more limited to those photons that passed very close to the focus. This rapid increase in 


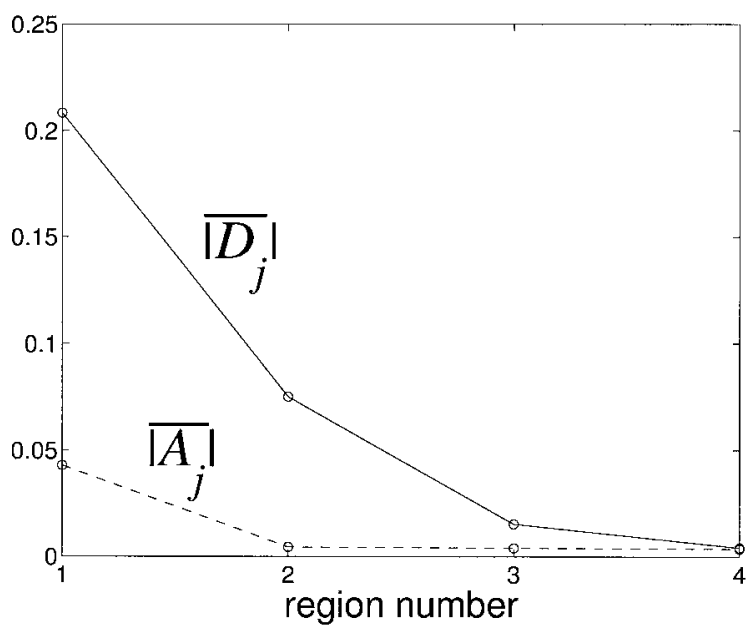

Fig. 8. Magnitude of the average value of $A_{j}=J_{1}\left(\left|\Phi_{j}\right|\right) \exp \left(i \delta_{j}\right)$ (dashed curve) and $D_{j}=\left[J_{0}(x)-1\right]$ (solid curve) for the four subsets of the detected photons.

the quantity $\left|\bar{D}_{j}\right|$ as the region number decreases is expected because the quantity $D_{j}$ is quadratic in the phase shifts. Thus, for the photons passing through region $4,\left|\bar{D}_{j}\right|$, which is approximately the same as the value of $\left|\bar{A}_{j}\right|$ (which was 0.003 for this same set of photons). However, for the set of photons passing through region $1,\left|\bar{D}_{j}\right|$, which is much larger than $\left|\bar{A}_{j}\right|, 0.04$ for this set. The value of the quantity $\left|\bar{A}_{j}\right|$ also changes by a large percentage between regions 1 and 2 in the plot of Fig. 8, suggesting that the significant contributions to the ac component may also be rather limited to those photons that passed through the focus. But, because only a small fraction (fewer than $10 \%$ ) of the photons in the simulation passed through region 1 , the cancellation effect, which diminishes the size of quantity $\left|\bar{A}_{j}\right|$ because of incoherent summation of ac contributions, may not be so great. In a physical experiment, many more photons are involved, and this will diminish the value of $\left|\bar{A}_{j}\right|$ for region 1 relative to those of other regions.

Because the size of its photon contribution is more sensitive to whether the corresponding photons passed through the focus, the dc shift of the API signal can potentially be used to improve the imaging resolution of API, which until now has made use primarily of the ac component amplitude in generating images.

\section{Summary}

We have theoretically analyzed the ultrasoundmodulated optical signal, or acoustophotonic imaging signal, of diffusely scattered light in the presence of a focused ultrasound beam in API, as processed by a new detection system that employs a photorefractive crystal. Equations (7) and (8) give, respectively, the dc and ac components of the API signal. The dc shift can be dominant, even though it is second order in the acoustically induced optical phase shifts. To obtain an understanding of the size of the acoustically in- duced phase shifts, we calculated the phase shifts for many photons that traveled through a diffusive medium in the presence of a realistic focused ultrasound field. The average magnitude of the phase shifts in the simulation was $\sim 12^{\circ}$, and the average magnitude for phase shifts of photons that passed through the focal region of the ultrasound field (corresponding to region 1 shown in Fig. 4) was $\sim 50^{\circ}$.

Quantities that characterize the dc and ac components of the API signal were also calculated for the detected photons in the simulation. The calculations confirm that the ac signal amplitude is dominated by the dc shift, as can be seen from Fig. 8. This is so because the photon contributions to the dc component add coherently, whereas the contributions to the ac component do not. We also found that the magnitude of the contributions to the dc shift effect is more sensitive to whether the corresponding photons passed through the ultrasound focal region than are the magnitudes of the ac contributions.

Therefore the dc shift can be used in place of or in conjunction with the API information provided by the ac component to improve the resolution of API. Whereas millimeter resolution can be achieved in a direction transverse to the ultrasound beam axis, which is set by the beam waist diameter, achieving the same resolution along the beam axis is a more involved problem. One way to improve axial resolution is to use very short ultrasound pulses, whose spatial length may be approximately the size of the focal region. By using the value of the dc shift instead of the ac signal amplitude to produce API images, one does not need to be concerned about ultrasound pulses' being too short to produce a steady-state ac signal; and the shortness of the resultant dc signal pulse is limited in practice only by the bandwidth of the photodetector (the typical duration of a dc signal pulse is approximately a few microseconds). Pulses are also well suited to the PRC detection systems and are safer in the clinical environment. The short-pulse method was demonstrated experimentally with the imaging of a centimeter-sized optical absorber. ${ }^{18}$ It provides an alternative to the method of frequencyswept ultrasound ${ }^{34}$ and other methods ${ }^{35}$ for improving axial resolution.

Future theoretical work will include calculating the amplitudes of components for API signals for various optical source and detector positions, whereas here we calculated phase shifts for generic photon paths. We are also interested in simulating the API signals obtained by use of ultrasound pulses and when absorbing inhomogeneities are present in the diffusive medium. The theoretical work will be compared with the results of experiments and used to help to configure an API system for clinical applications.

This research was supported by the Center for Subsurface Sensing and Imaging Systems, under the Engineering Research Centers Program of the National Science Foundation (award EEC-9986821). 


\section{References}

1. V. V. Tuchin, ed., Handbook of Optical Biomedical Diagnostics (SPIE, 2002).

2. D. A. Boas, D. H. Brooks, E. L. Miller, C. A. DiMarzio, M. Kilmer, R. J. Gaudette, and Q. Zhang, "Imaging the body with diffuse optical tomography," in IEEE Signal Process. Mag. 18, 57-75 (2001)

3. J. A. Moon, R. Mahon, M. D. Duncan, and J. Reintjes, "Resolution limits for imaging through turbid media with diffuse light," Opt. Lett. 18, 1591-1593 (1993).

4. F. A. Marks, H. W. Tomlinson, and G. W. Brooksby, "A comprehensive approach to breast cancer detection using light: photon localization by ultrasound modulation and tissue characterization by spectral discrimination," in Photon Migration and Imaging in Random Media and Tissues, B. Chance and R. A. Alfano, eds., Proc. SPIE 1888, 500-510 (1993).

5. G. D. Mahan, W. E. Engler, J. J. Tiemann, and E. G. Uzgiris, "Ultrasonic tagging of light: theory," Proc. Natl. Acad. Sci. USA 95, 14,015-14,019 (1998).

6. L.-H. V. Wang, S. L. Jacques, and X.-M. Zhao, "Continuouswave ultrasonic modulation of scattered laser light to image objects in turbid media," Opt. Lett. 20, 629-631 (1995).

7. L.-H. V. Wang and X.-M. Zhao, "Ultrasound-modulated optical tomography of absorbing objects buried in dense tissuesimulating turbid media," Appl. Opt. 36, 7277-7282 (1997).

8. M. Kempe, M. Larionov, D. Zaslavsky, and A. Z. Genack, "Acousto-optic tomography with multiply scattered light," J. Opt. Soc. Am. A 14, 1151-1158 (1997).

9. S. Leveque, A. C. Boccara, M. Lebec, and H. Saint-Jalmes, "Ultrasonic tagging of photon paths in scattering media: parallel speckle modulation processing," Opt. Lett. 24, 181-183 (1999).

10. C. A. DiMarzio, R. J. Gaudette, and T. J. Gaudette, "A new imaging technique combining diffusive photon density waves and focused ultrasound," in Optical Tomography and Spectroscopy of Tissue III, B. Chance, R. A. Alfano, and B. J. Tromberg, eds., Proc. SPIE 3597, 376-384 (1999).

11. S. Leveque, J. Selb, L. Pottier, and A. C. Boccara, "In situ local tissue characterization and imaging by backscattering acousto-optic imaging," Opt. Commun. 196, 127-131 (2001).

12. D. Dolfi and F. Micheron, "Imaging process and system for transillumination with photon frequency marking," U.S. patent, 5,174,298 (29 December 1992).

13. A. Lev and B. Sfez, "In vivo demonstration of the ultrasoundmodulated light technique,” J. Opt. Soc. Am. A 20, 2347-2354 (2003).

14. L.-H. V. Wang, "Mechanisms of ultrasonic modulation of multiply scattered coherent light: an analytic model," Phys. Rev. Lett. 87, 043903 (2001).

15. L.-H. V. Wang, "Mechanisms of ultrasonic modulation of multiply scattered coherent light: a Monte Carlo model," Opt. Lett. 26, 1191-1193 (2001).

16. S. Sakadžić and L.-H. V. Wang, "Ultrasonic modulation of multiply scattered coherent light: an analytical model for anisotropically scattering media," Phys. Rev. E 66, 026603 (2002).

17. G. Yao and L.-H. V. Wang, "Signal dependence and noise source in ultrasound-modulated optical tomography," Appl. Opt. 43, 1320-1326 (2004).

18. T. W. Murray, L. Sui, G. Maguluri, R. A. Roy, A. Nieva, F. Blonigen, and C. A. DiMarzio, "Detection of ultrasoundmodulated photons in diffuse media using the photorefractive effect," Opt. Lett. 29, 2509-2511 (2004).

19. R. K. Ing and J. P. Monchalin, "Broadband optical detection of ultrasound by two-wave mixing in a photorefractive crystal," Appl. Phys. Lett. 59, 3233-3235 (1991).

20. A. Blouin and J. P. Monchalin, "Detection of ultrasonic motion of a scattering surface by two-wave mixing in a photorefractive GaAs crystal,” Appl. Phys. Lett. 65, 932-934 (1994).

21. P. Delaye, A. Blouin, D. Drolet, L.-A. de Montmorillon, G. Roosen, and J.-P. Monchalin, "Detection of ultrasonic motion of a scattering surface by photorefractive InP:Fe under an applied dc field," J. Opt. Soc. Am. B 14, 1723-1734 (1997).

22. A. Nieva, S. Manneville, D. A. Boas, R. Roy, and C. A. DiMarzio, "Monte Carlo simulations in acousto-photonic imaging," in Digest of Topical Meeting on Biomedical Optics (Optical Society of America, 2002).

23. M. Born and E. Wolf, Principles of Optics, 6th ed. (Pergamon, 1987)

24. W. Leutz and G. Maret, "Ultrasonic modulation of multiply scattered light," Physica B 204, 14-19 (1995).

25. P. Delaye, L.-A. de Montmorillon, and G. Roosen, "Transmission of time modulated optical signals through an absorbing photorefractive crystal," Opt. Commun. 118, 154-164 (1995).

26. R. D. Guenther, Modern Optics (Wiley, 1990).

27. M. Hamilton and D. Blackstock, Nonlinear Acoustics: Theory and Applications (Academic, 1998).

28. I. M. Hallaj and R. O. Cleveland, "FDTD simulation of finite amplitude pressure and temperature fields for biomedical ultrasound," J. Acoust. Soc. Am. 105, L7-L12 (1999).

29. K. Yosioka and Y. Kawasima, "Acoustic radiation pressure on a compressible sphere," Acustica 5, 167-173 (1955).

30. W. F. Cheong, S. A. Prahl, and A. J. Welch, "A review of the optical properties of biological tissues," IEEE J. Quantum Electron. 26, 2166-2185 (1990).

31. V. G. Peters, D. R. Wyman, M. S. Patterson, and G. L. Frank, "Optical properties of normal and diseased human breast tissues in the visible and near infrared," Phys. Med. Biol. 35, 1317-1334 (1990).

32. S. A. Prahl, M. Keijzer, S. L. Jacques, and A. J. Welch, "A Monte Carlo model for light propagation in tissue," in Dosimetry of Laser Radiation in Medicine and Biology, Vol. IS5 of SPIE Institute Series (SPIE, 1989), pp. 102-111.

33. H. C. van de Hulst, Multiple Light Scattering (Academic, 1980), Vol. 2.

34. L.-H. V. Wang and G. Ku, "Frequency-swept ultrasoundmodulated optical tomography of scattering media," Opt. Lett. 23, 975-977 (1998).

35. A. Lev and B. G. Sfez, "Pulsed ultrasound-modulated light tomography," Opt. Lett. 28, 1549-1551 (2003). 